PROCEEDINGS OF THE

AMERICAN MATHEMATICAL SOCIETY

Volume 136, Number 11, November 2008, Pages 3963-3973

S 0002-9939(08)09392-1

Article electronically published on June 9, 2008

\title{
PARAMETRIC REPRESENTATION AND ASYMPTOTIC STARLIKENESS IN $\mathbb{C}^{n}$
}

\author{
IAN GRAHAM, HIDETAKA HAMADA, GABRIELA KOHR, AND MIRELA KOHR
}

(Communicated by Mei-Chi Shaw)

\begin{abstract}
In this paper we consider the notion of asymptotic starlikeness in the Euclidean space $\mathbb{C}^{n}$. In the case of the maximum norm, asymptotic starlikeness was introduced by Poreda. We have modified his definition slightly, adding a boundedness condition. We prove that the notion of parametric representation which arises in Loewner theory can be characterized in terms of asymptotic starlikeness; i.e. they are equivalent notions. (A regularity assumption of Poreda is not needed.) In particular, starlike mappings and spirallike mappings of type $\alpha \in(-\pi / 2, \pi / 2)$ are asymptotically starlike. Therefore this notion is a natural generalization of starlikeness. However, we give an example of a spirallike mapping with respect to a linear operator which is not asymptotically starlike. In the case of one complex variable, any function in the class $S$ is asymptotically starlike; however, in dimension $n \geq 2$ this is no longer true.
\end{abstract}

\section{INTRODUCTION}

Let $\mathbb{C}^{n}$ denote the space of $n$ complex variables $z=\left(z_{1}, \ldots, z_{n}\right)$ with the Euclidean inner product $\langle z, w\rangle=\sum_{j=1}^{n} z_{j} \bar{w}_{j}$ and the Euclidean norm $\|z\|=\langle z, z\rangle^{1 / 2}$. The open ball $\left\{z \in \mathbb{C}^{n}:\|z\|<r\right\}$ is denoted by $B_{r}^{n}$ and the unit ball $B_{1}^{n}$ is denoted by $B^{n}$. The closed ball $\left\{z \in \mathbb{C}^{n}:\|z\| \leq r\right\}$ is denoted by $\bar{B}_{r}^{n}$. In the case of one complex variable, $B^{1}$ is denoted by $U$.

Let $L\left(\mathbb{C}^{n}, \mathbb{C}^{m}\right)$ denote the space of linear and continuous operators from $\mathbb{C}^{n}$ into $\mathbb{C}^{m}$ with the standard operator norm and let $I_{n}$ be the identity in $L\left(\mathbb{C}^{n}, \mathbb{C}^{n}\right)$. If $\Omega$ is a domain in $\mathbb{C}^{n}$, let $H(\Omega)$ be the set of holomorphic mappings from $\Omega$ into $\mathbb{C}^{n}$. If $\Omega$ is a domain in $\mathbb{C}^{n}$ which contains the origin and $f \in H(\Omega)$, we say that $f$ is normalized if $f(0)=0$ and $D f(0)=I_{n}$. Let $S\left(B^{n}\right)$ be the set of normalized biholomorphic mappings on $B^{n}$. In the case of one complex variable, the set $S\left(B^{1}\right)$ is denoted by $S$. Also let $S^{*}\left(B^{n}\right)$ be the subset of $S\left(B^{n}\right)$ consisting of starlike mappings on $B^{n}$.

Received by the editors December 6, 2006, and, in revised form, October 15, 2007.

2000 Mathematics Subject Classification. Primary 32H02; Secondary 30C45.

Key words and phrases. Asymptotic starlikeness, biholomorphic mapping, Loewner chain, parametric representation, spirallike mapping, starlike mapping.

The first author was partially supported by the Natural Sciences and Engineering Research Council of Canada under Grant A9221.

The second author was partially supported by Grant-in-Aid for Scientific Research (C) no. 19540205 from the Japan Society for the Promotion of Science, 2007.

The third and fourth authors were partially supported by the Romanian Ministry of Education and Research, CNCSIS Grant type A, 1472/2007.

(C)2008 American Mathematical Society Reverts to public domain 28 years from publication 
If $f \in H\left(B^{n}\right)$, we say that $f$ is locally biholomorphic on $B^{n}$ if $J_{f}(z) \neq 0, z \in B^{n}$, where $J_{f}(z)=\operatorname{det} D f(z)$ and $D f(z)$ is the complex Jacobian matrix of $f$ at $z$. Let $\mathcal{L} S_{n}$ be the set of normalized locally biholomorphic mappings on $B^{n}$.

If $f, g \in H\left(B^{n}\right)$, we say that $f$ is subordinate to $g(f \prec g)$ if there exists a Schwarz mapping $v$ (i.e. $v \in H\left(B^{n}\right)$ and $\|v(z)\| \leq\|z\|, z \in B^{n}$ ) such that $f=g \circ v$.

Definition 1.1. A mapping $f: B^{n} \times[0, \infty) \rightarrow \mathbb{C}^{n}$ is called a univalent subordination chain if $f(\cdot, t)$ is biholomorphic on $B^{n}, f(0, t)=0$ for $t \geq 0$, and $f(\cdot, s) \prec f(\cdot, t)$, $0 \leq s \leq t<\infty$. If $D f(0, t)=e^{t} I_{n}$ for $t \geq 0$, we say that $f(z, t)$ is a Loewner chain (or a normalized univalent subordination chain).

The above subordination condition is equivalent to the existence of a unique Schwarz mapping $v=v(z, s, t)$, called the transition mapping associated with $f(z, t)$, such that $f(z, s)=f(v(z, s, t), t), z \in B^{n}, t \geq s \geq 0$.

The following class of mappings in $H\left(B^{n}\right)$ plays the role of the Carathéodory class in $\mathbb{C}^{n}$ :

$$
\mathcal{M}=\left\{h \in H\left(B^{n}\right): h(0)=0, D h(0)=I_{n}, \Re\langle h(z), z\rangle>0, z \in B^{n} \backslash\{0\}\right\} .
$$

We remark that the above class plays a key role in the study of Loewner chains and the Loewner differential equation in dimension $n \geq 2$ (see [4, 7], 8], 13]).

Definition 1.2 (see [4, 7]; cf. [15]). We say that a normalized mapping $f \in$ $H\left(B^{n}\right)$ has parametric representation if there exists a mapping $h: B^{n} \times[0, \infty) \rightarrow \mathbb{C}^{n}$ such that $h(\cdot, t) \in \mathcal{M}$ for $t \in[0, \infty), h(z, \cdot)$ is measurable on $[0, \infty)$ for $z \in B^{n}$, and $f(z)=\lim _{t \rightarrow \infty} e^{t} v(z, t)$ locally uniformly on $B^{n}$, where $v=v(z, t)$ is the unique locally absolutely continuous solution on $[0, \infty)$ of the initial value problem

$$
\partial v / \partial t=-h(v, t) \quad \text { a.e. } t \geq 0, v(z, 0)=z,
$$

for all $z \in B^{n}$.

Remark 1.3. The above condition is equivalent to the fact that there exists a Loewner chain $f(z, t)$ such that $\left\{e^{-t} f(\cdot, t)\right\}_{t \geq 0}$ is a normal family on $B^{n}$ and $f(z)=f(z, 0), z \in B^{n}$ (see [4], 7], [8]; cf. [16]).

We denote by $S^{0}\left(B^{n}\right)$ the class of mappings which have parametric representation. If $n=1, S^{0}(U)=S$ (see [14]), but $S^{0}\left(B^{n}\right) \varsubsetneqq S\left(B^{n}\right)$ for $n \geq 2$ (see [4] and [7]; cf. 15]). However, important subclasses of $S\left(B^{n}\right)$ are also subclasses of $S^{0}\left(B^{n}\right)$. For example, $S^{*}\left(B^{n}\right) \varsubsetneqq S^{0}\left(B^{n}\right)$. For applications, see [4, 6], 7], 8], 9].

Definition 1.4 (cf. [21]). Let $A \in L\left(\mathbb{C}^{n}, \mathbb{C}^{n}\right)$ be such that $m(A)>0$, where

$$
m(A)=\min \{\Re\langle A(z), z\rangle:\|z\|=1\} .
$$

Also let $\Omega$ be a domain in $\mathbb{C}^{n}$ which contains the origin. We say that $\Omega$ is spirallike with respect to $A$ if $e^{-t A}(w) \in \Omega$, for all $w \in \Omega$ and $t \geq 0$.

A mapping $f \in S\left(B^{n}\right)$ is called spirallike with respect to $A$ if $f\left(B^{n}\right)$ is a spirallike domain with respect to $A$.

Remark 1.5. Let $f \in \mathcal{L} S_{n}$. It is well known that $f$ is spirallike with respect to $A$ if and only if (see [21, Theorem 11]; compare with [10, Definition 3, Theorem 4])

$$
\Re\left\langle[D f(z)]^{-1} A f(z), z\right\rangle>0, z \in B^{n} \backslash\{0\} .
$$

A mapping $f$ is called spirallike of type $\alpha \in(-\pi / 2, \pi / 2)$ if $f$ is spirallike with respect to $A=e^{-i \alpha} I_{n}$ (see [11; cf. 21]). Hence $f \in S^{*}\left(B^{n}\right)$ if and only if $f$ is spirallike of type zero. 
Hamada and Kohr [11] showed that spirallikeness of type $\alpha$ has the following characterization in terms of Loewner chains: $f \in \mathcal{L} S_{n}$ is spirallike of type $\alpha$ if and only if $f(z, t)=e^{(1-i a) t} f\left(e^{i a t} z\right)$ is a Loewner chain, where $a=\tan \alpha$. In particular, $f \in S^{*}\left(B^{n}\right)$ if and only if $f(z, t)=e^{t} f(z)$ is a Loewner chain.

The study of the class $S\left(B^{n}\right)$, which is the analog of the class $S$ in several complex variables, was first suggested by H. Cartan (see [1]). He studied biholomorphic mappings on the unit polydisc in $\mathbb{C}^{2}$ and gave a counterexample which shows that the growth theorem for the class $S$ fails in dimension $n \geq 2$. Cartan also suggested that particular subclasses of normalized biholomorphic mappings, such as the starlike and convex mappings, should be singled out for further development. Indeed, many of the results in univalent function theory have extensions to higher dimensions for these classes of mappings. Suffridge (see [19], 20], 21]) obtained analytical characterizations of starlikeness and convexity on the unit ball in $\mathbb{C}^{n}$ as well as in complex Banach spaces. Gong and others (see [3], 7] and the references therein, [12]) studied in detail growth and covering results for normalized starlike and convex mappings in several complex variables. Also, Hamada and Kohr [11] obtained the growth theorem for spirallike mappings of type $\alpha$. Further results concerning spirallike mappings with respect to a positive linear operator can be found in [2], 10, 17, [18, 21]. These results show that the geometric properties of $f\left(B^{n}\right)$ for $f \in S\left(B^{n}\right)$ play an important role for the growth theorem. Another class of mappings for which the growth theorem holds is the class $S^{0}\left(B^{n}\right)$ (see 4, [7, 8, 9] for the Euclidean case and the case of a general norm, and [15, [16] for the case of the polydisc).

A natural question arises:

Question 1.6. What is a geometric characterization of $f\left(B^{n}\right)$ for $f \in S^{0}\left(B^{n}\right)$ ?

The main goal of our paper is to give an answer to this question.

\section{Preliminaries}

In this section we introduce another notion which involves differential equations, and which may be regarded as giving geometric characterizations of certain domains in $\mathbb{C}^{n}$. We then consider the relation between this notion and Loewner chains.

Definition 2.1. Let $\Omega \subseteq \mathbb{C}^{n}$ be a domain which contains the origin. We say that $\Omega$ is asymptotically starlike if there exists a mapping $Q=Q(z, t): \Omega \times[0, \infty) \rightarrow \mathbb{C}^{n}$ which satisfies the following properties:

(i) $Q(\cdot, t)$ is a holomorphic mapping on $\Omega, Q(0, t)=0, D Q(0, t)=I_{n}, t \geq 0$, and the family $\{Q(\cdot, t)\}_{t \geq 0}$ is locally uniformly bounded on $\Omega$.

(ii) $Q(z, \cdot)$ is measurable on $[0, \infty)$ for all $z \in \Omega$.

(iii) The initial value problem

$$
\frac{\partial w}{\partial t}=-Q(w, t) \quad \text { a.e. } t \geq s, w(z, s, s)=z,
$$

has a unique solution $w=w(z, s, t)$ for each $z \in \Omega$ and $s \geq 0$, such that $w(\cdot, s, t)$ is a holomorphic mapping of $\Omega$ into $\Omega$ for $t \geq s, w(z, s, \cdot)$ is locally absolutely continuous on $[s, \infty)$ locally uniformly with respect to $z \in \Omega$ for $s \geq 0$, and $\lim _{t \rightarrow \infty} e^{t} w(z, 0, t)=z$ locally uniformly on $\Omega$. 
Definition 2.2. Let $f: B^{n} \rightarrow \mathbb{C}^{n}$ be a normalized holomorphic mapping. We say that $f$ is asymptotically starlike if $f$ is biholomorphic on $B^{n}$ and $f\left(B^{n}\right)$ is an asymptotically starlike domain.

Remark 2.3. (i) In the case of the maximum norm, the notion of asymptotic starlikeness was introduced by Poreda [16. His definition does not require the normality assumption related to the family $\{Q(\cdot, t)\}_{t \geq 0}$. However, as we will see in the proof of the main result of our paper, this assumption is natural. Poreda proved that any mapping with parametric representation on the unit polydisc in $\mathbb{C}^{n}$ is asymptotically starlike. On the other hand, he imposed a strong assumption related to the differentiability of the solution $w(z, s, t)$ of the initial value problem (2.1) at the point $t=s$, in order to deduce that such an asymptotically starlike mapping also has parametric representation. This assumption is not necessary in our case.

(ii) If $Q(\cdot, t) \equiv I_{n}$ in Definition 2.1, then $\Omega$ is starlike with respect to zero.

Indeed, the initial value problem (2.1) has the unique solution $w(z, s, t)=e^{s-t} z$ such that $w(z, s, t) \in \Omega$ for $z \in \Omega$ and $t \geq s$.

(iii) If $\Omega \subseteq \mathbb{C}^{n}$ is a starlike domain with respect to zero, then $\Omega$ is asymptotically starlike.

Again, it suffices to consider $Q(\cdot, t) \equiv I_{n}$ in Definition 2.1.

In this paper we prove the following geometric interpretation for the notion of parametric representation: a mapping $f \in S\left(B^{n}\right)$ is asymptotically starlike if and only if $f$ has parametric representation. We also deduce that any spirallike mapping of type $\alpha$ is asymptotically starlike. However, we give an example which shows that in dimension $n \geq 2$ there exist spirallike mappings with respect to a linear operator which are not asymptotically starlike.

\section{PARAMETRIC REPRESENTATION AND ASYMPtotiC STARLIKENESS}

In this section we prove the following result (compare with [16, Theorems 1, 2]).

Theorem 3.1. Let $f \in S\left(B^{n}\right)$. Then $f \in S^{0}\left(B^{n}\right)$ if and only if $f$ is asymptotically starlike.

Proof. Let $\Omega=f\left(B^{n}\right)$. First, we assume that $f \in S^{0}\left(B^{n}\right)$. (In the first part of the proof, we shall use arguments similar to those in the proof of [16, Theorem 1].) Since $f \in S^{0}\left(B^{n}\right)$, there exists a mapping $h=h(z, t): B^{n} \times[0, \infty) \rightarrow \mathbb{C}^{n}$, which satisfies the conditions in Definition 1.2, such that $\lim _{t \rightarrow \infty} e^{t} v(z, t)=f(z)$ locally uniformly on $B^{n}$, where $v=v(z, t)$ is the unique locally absolutely continuous solution on $[0, \infty)$ of the initial value problem

$$
\frac{\partial v}{\partial t}=-h(v, t) \quad \text { a.e. } \quad t \geq 0, v(z, 0)=z .
$$

Then $v(\cdot, t)$ is a biholomorphic Schwarz mapping and $D v(0, t)=e^{-t} I_{n}$ by [13, Theorem 2.1]. On the other hand, since $f \in S^{0}\left(B^{n}\right)$, there exists a Loewner chain $f(z, t)$ such that $\left\{e^{-t} f(\cdot, t)\right\}_{t \geq 0}$ is a normal family on $B^{n}$ and $f=f(\cdot, 0)$. Also let $V(z, s, t)$ be the transition mapping associated with $f(z, t)$. Then $V(\cdot, s, t)$ is a biholomorphic Schwarz mapping such that $D V(0, s, t)=e^{s-t} I_{n}$ and $V(z, s, \cdot)$ is Lipschitz continuous on $[s, \infty)$ locally uniformly with respect to $z \in B^{n}$ for $s \geq 0$, and for each $s \geq 0$,

$$
f(z, s)=\lim _{t \rightarrow \infty} e^{t} V(z, s, t)
$$


where the above limit holds locally uniformly on $B^{n}$ (see [8] and 9]; cf. [15]). Moreover, $V(z, s, t)$ satisfies the initial value problem

$$
\frac{\partial V}{\partial t}=-h(V, t) \quad \text { a.e. } \quad t \geq s, V(z, s, s)=z,
$$

for all $z \in B^{n}$ and $s \geq 0$ (see [4] and [8]; see also [7]). In view of (3.1) and (3.3), we deduce that $v(z, t)=V(z, 0, t)$ for $z \in B^{n}$ and $t \geq 0$.

Next, let $Q: \Omega \times[0, \infty) \rightarrow \mathbb{C}^{n}$ be given by

$$
Q(w, t)=D f(z) h(z, t), \quad w=f(z) \in \Omega, \quad t \geq 0 .
$$

Then $Q(\cdot, t) \in H(\Omega), Q(0, t)=0$ and $D Q(0, t)=I_{n}$ for $t \geq 0$. Clearly $Q(w, \cdot)$ is measurable on $[0, \infty)$ for $w \in \Omega$ in view of the measurability of $h(z, \cdot)$ on $[0, \infty)$. On the other hand, since $\mathcal{M}$ is a compact subset of $H\left(B^{n}\right)$ (see [4]) and $h(\cdot, t) \in \mathcal{M}$ for $t \geq 0$, it follows that for each $r \in(0,1)$ there exists $M=M(r)>0$ such that

$$
\|h(z, t)\| \leq M(r),\|z\| \leq r, t \geq 0 .
$$

Since $f$ is bounded on $\bar{B}_{r}^{n}$, we deduce that for each $r \in(0,1)$ there exists $M^{*}=$ $M^{*}(r)>0$ such that

$$
\|Q(f(z), t)\| \leq M^{*}(r),\|z\| \leq r, t \geq 0 .
$$

Hence $\{Q(\cdot, t)\}_{t \geq 0}$ is locally uniformly bounded on $\Omega$.

Let $\nu(w, s, t)=f(V(z, s, t))$ for $w=f(z) \in f\left(B^{n}\right)$ and $t \geq s \geq 0$. Then $\nu(\cdot, s, t)$ is a holomorphic mapping of $\Omega$ into $\Omega, \nu(0, s, t)=0$ for $t \geq s \geq 0$, and $\nu(w, s, \cdot)$ is locally absolutely continuous on $[s, \infty)$ locally uniformly with respect to $w \in \Omega$, $\nu(w, s, s)=w$ for $w \in \Omega$ and $s \geq 0$. A short computation yields that

$$
\frac{\partial \nu}{\partial t}=-D f(V(z, s, t)) h(V(z, s, t), t)=-Q(f(V(z, s, t)), t),
$$

for almost all $t \geq s$ and for all $z \in B^{n}$. Therefore, $\nu(w, s, t)$ is a solution of the initial value problem

$$
\frac{\partial \nu}{\partial t}=-Q(\nu, t) \quad \text { a.e. } \quad t \geq s, \nu(w, s, s)=w,
$$

for all $w \in \Omega$ and $s \geq 0$. By the uniqueness of solutions to the initial value problem (3.3), we deduce that (3.4) has the unique locally absolutely continuous solution $\nu=\nu(w, s, t)=f(V(z, s, t))$ on $[s, \infty)$ for $w=f(z) \in f\left(B^{n}\right)$ and $t \geq s \geq 0$.

Moreover, taking into account the relation (3.2), the normalization of $f$, the fact that $V(z, 0, t) \rightarrow 0$ locally uniformly on $B^{n}$ as $t \rightarrow \infty$, and $D V(0,0, t)=e^{-t} I_{n}$, we deduce that

$$
\lim _{t \rightarrow \infty} e^{t} \nu(w, 0, t)=\lim _{t \rightarrow \infty} e^{t} f\left(V\left(f^{-1}(w), 0, t\right)\right)=w
$$

locally uniformly on $\Omega$.

Therefore $\Omega=f\left(B^{n}\right)$ is asymptotically starlike. Since $f$ is biholomorphic on $B^{n}$, it follows that $f$ is asymptotically starlike, as desired.

Conversely, assume that $f$ is asymptotically starlike. Let $Q: \Omega \times[0, \infty) \rightarrow \mathbb{C}^{n}$ be a mapping that satisfies the assumptions of Definition 2.1. Also let $\nu=\nu(f(z), s, t)$ be the unique solution of the initial value problem

$$
\frac{\partial \nu}{\partial t}=-Q(\nu, t) \quad \text { a.e. } \quad t \geq s, \nu(f(z), s, s)=f(z),
$$


for all $z \in B^{n}$ and $s \geq 0$. Then $\nu(\cdot, s, t)$ is a holomorphic mapping of $f\left(B^{n}\right)$ into $f\left(B^{n}\right), \nu(0, s, t)=0$ for $t \geq s$ and $\nu(f(z), s, \cdot)$ is locally absolutely continuous on $[s, \infty)$ locally uniformly with respect to $f(z) \in f\left(B^{n}\right)$. Moreover, we know that

$$
\lim _{t \rightarrow \infty} e^{t} \nu(w, 0, t)=w
$$

locally uniformly on $\Omega=f\left(B^{n}\right)$.

Let $v=v(z, s, t)$ be defined by

$$
v(z, s, t)=f^{-1}(\nu(f(z), s, t)), z \in B^{n}, t \geq s .
$$

Then $v(\cdot, s, t)$ is a Schwarz mapping. Moreover, $v(z, s, \cdot)$ is locally absolutely continuous on $[s, \infty)$ locally uniformly with respect to $z \in B^{n}$ and $v(z, s, s)=z$ for $z \in B^{n}$. In view of (3.6) and the fact that $f$ is normalized, we deduce that

$$
\lim _{t \rightarrow \infty} e^{t} v(z, 0, t)=f(z)
$$

and the above limit holds locally uniformly on $B^{n}$.

Next, let $h=h(z, t): B^{n} \times[0, \infty) \rightarrow \mathbb{C}^{n}$ be given by

$$
h(z, t)=[D f(z)]^{-1} Q(f(z), t), z \in B^{n}, t \geq 0 .
$$

Then $h(\cdot, t) \in H\left(B^{n}\right)$ for $t \geq 0, h(z, \cdot)$ is measurable on $[0, \infty)$ for $z \in B^{n}$, and since $Q(\cdot, t)$ and $f$ are normalized, it follows that $h(\cdot, t)$ is normalized too for $t \geq 0$. Since $\{Q(\cdot, t)\}_{t \geq 0}$ is a locally uniformly bounded family on $f\left(B^{n}\right)$, it follows that for each $r \in(0,1)$, there exists $L=L(r)>0$ such that

$$
\|Q(f(z), t)\| \leq L(r),\|z\| \leq r, t \geq 0 .
$$

Further, since $f$ is biholomorphic on $B^{n},[D f(z)]^{-1}$ is uniformly bounded on each closed ball $\bar{B}_{r}^{n}$, and hence for each $r \in(0,1)$, there exists $L^{*}=L^{*}(r)>0$ such that

$$
\|h(z, t)\| \leq L^{*}(r),\|z\| \leq r, t \geq 0 .
$$

Next, taking into account (3.5), we deduce that

$$
\frac{\partial v}{\partial t}=-[D f(v(z, s, t))]^{-1} Q(f(v(z, s, t)), t)=-h(v(z, s, t), t),
$$

for almost all $t \geq s$ and for all $z \in B^{n}$. Hence $v(z, s, t)$ is a solution of the initial value problem

$$
\frac{\partial v}{\partial t}=-h(v, t) \quad \text { a.e. } \quad t \geq s, v(z, s, s)=z,
$$

for all $s \geq 0$ and $z \in B^{n}$. By the uniqueness of solutions to (3.5), we deduce that $v(z, s, t)=f^{-1}(\nu(f(z), s, t))$ is the unique locally absolutely continuous solution of (3.9). Moreover, in view of (3.8) and (3.9) we deduce that

$$
\left\|v\left(z, s, t_{1}\right)-v\left(z, s, t_{2}\right)\right\|=\left\|\int_{t_{1}}^{t_{2}} h(v(z, s, t), t) d t\right\| \leq L^{*}(r)\left(t_{2}-t_{1}\right),
$$

for $\|z\| \leq r<1$ and $s \leq t_{1} \leq t_{2}<\infty$. Hence $v(z, s, \cdot)$ is Lipschitz continuous on $[s, \infty)$ locally uniformly with respect to $z \in B^{n}$.

We next prove that $v(z, \cdot, t)$ is also Lipschitz continuous on $[0, t]$ locally uniformly with respect to $z \in B^{n}$. To this end, we first note that the uniqueness of solutions to the initial value problem (3.9) easily yields the following semigroup property:

$$
v(z, s, u)=v(v(z, s, t), t, u), z \in B^{n}, 0 \leq s \leq t \leq u<\infty .
$$


Next, fix $t \geq 0$ and let $0 \leq s_{1} \leq s_{2} \leq t$. Since

$$
\left\|v\left(z, s_{2}, t\right)-v\left(z^{\prime}, s_{2}, t\right)\right\| \leq\left\|z-z^{\prime}\right\| \int_{0}^{1}\left\|D v\left((1-\tau) z+\tau z^{\prime}, s_{2}, t\right)\right\| d \tau,
$$

it follows in view of the Cauchy integral formula for holomorphic mappings and the relation $\left\|v\left(z, s_{2}, t\right)\right\| \leq\|z\|$ that for each $r \in(0,1)$ there exists $K=K(r)>0$ such that

$$
\left\|v\left(z, s_{2}, t\right)-v\left(z^{\prime}, s_{2}, t\right)\right\| \leq K(r)\left\|z-z^{\prime}\right\|,\|z\| \leq r,\left\|z^{\prime}\right\| \leq r .
$$

Replacing $z^{\prime}$ by $v\left(z, s_{1}, s_{2}\right)$ in the above inequality and using (3.10) and (3.11), we deduce for each $z \in \bar{B}_{r}^{n}$ that

$$
\left\|v\left(z, s_{1}, t\right)-v\left(z, s_{2}, t\right)\right\| \leq K(r)\left\|z-v\left(z, s_{1}, s_{2}\right)\right\| \leq K(r) L^{*}(r)\left(s_{2}-s_{1}\right) .
$$

Hence $v(z, \cdot, t)$ is Lipschitz continuous on $[0, t]$ locally uniformly with respect to $z \in B^{n}$, as claimed.

Further, using the Lipschitz continuity of $v(z, s, \cdot)$ locally uniformly with respect to $z \in B^{n}$ and Vitali's theorem for holomorphic mappings in several complex variables, we deduce that $\frac{\partial v}{\partial t}(\cdot, s, t)$ exists and is a holomorphic mapping on $B^{n}$ for almost all $t \geq s$.

Next, fix $\eta>0$ and let $x=v(z, s, t)$. From (3.11) we have

$$
\begin{gathered}
\frac{v(z, s, t+\eta)-v(z, s, t)}{\eta}=\frac{v(v(z, s, t), t, t+\eta)-v(z, s, t)}{\eta} \\
=\frac{v(x, t, t+\eta)-x}{\eta}, z \in B^{n}, t \geq s \geq 0 .
\end{gathered}
$$

Since the limit of the left-hand side of (3.12) exists for $\eta \rightarrow 0+$ and is equal to $\frac{\partial v}{\partial t}(z, s, t)$ for almost all $t \geq s$, we deduce that the limit of the right-hand side of (3.12) also exists for $\eta \rightarrow 0+$. Using the relations (3.9) and (3.12), we deduce except for a set of measure zero in $t \geq s$ that

$$
\lim _{\eta \rightarrow 0+} \frac{x-v(x, t, t+\eta)}{\eta}=h(v(z, s, t), t), \forall z \in B^{n} .
$$

On the other hand, since $\|v(x, t, t+\eta)\| \leq\|x\|$ it follows in view of the above relation that

$$
\Re\langle h(v(z, s, t), t), v(z, s, t)\rangle \geq 0, \quad \text { a.e. } \quad t \geq s, \quad \forall z \in B^{n} .
$$

Let $\mathbb{Q}_{+}$be the set of nonnegative rational numbers and let $\lambda$ be the usual Lebesgue measure in $\mathbb{R}$. Then for each $s_{k} \in \mathbb{Q}_{+}$, there exists $E_{k} \subset\left[s_{k}, \infty\right)$ such that $\lambda\left(E_{k}\right)=0$ and

$$
\Re\left\langle h\left(v\left(z, s_{k}, t\right), t\right), v\left(z, s_{k}, t\right)\right\rangle \geq 0, \quad t \in\left[s_{k}, \infty\right) \backslash E_{k},
$$

by (3.13). Let $E=\bigcup_{k \in \mathbb{N}} E_{k}$. Then $\lambda(E)=0$ and if $t \in[0, \infty) \backslash E$ is fixed, we deduce in view of (3.14) that

$$
\Re\left\langle h\left(v\left(z, s_{k}, t\right), t\right), v\left(z, s_{k}, t\right)\right\rangle \geq 0, z \in B^{n}, s_{k} \in \mathbb{Q}_{+}, s_{k} \leq t, k \in \mathbb{N} .
$$

Further, letting $\left\{s_{\nu(k)}\right\}_{k \in \mathbb{N}} \subset \mathbb{Q}_{+}, s_{\nu(k)} \nearrow t$ in the above relation and using the fact that $v(z, \cdot, t)$ is continuous on $[0, t]$, we conclude that $\Re\langle h(z, t), z\rangle \geq 0, z \in B^{n}$. Since $h(0, t)=0$ and $D h(0, t)=I_{n}$, it follows in view of the minimum principle for harmonic functions that (see also [20, Lemma 3])

$$
\Re\langle h(z, t), z\rangle>0, z \in B^{n} \backslash\{0\} .
$$


Hence $h(\cdot, t) \in \mathcal{M}$ for $t \in[0, \infty) \backslash E$. Finally, let $\tilde{h}=\tilde{h}(z, t): B^{n} \times[0, \infty) \rightarrow \mathbb{C}^{n}$ be given by

$$
\tilde{h}(z, t)= \begin{cases}h(z, t), & z \in B^{n}, t \in[0, \infty) \backslash E \\ z, & z \in B^{n}, t \in E\end{cases}
$$

Then $\tilde{h}(\cdot, t) \in \mathcal{M}$ for $t \geq 0$. Concluding the above arguments, we deduce in view of the relations (3.7) and (3.9) that $f \in S^{0}\left(B^{n}\right)$. This completes the proof.

Remark 3.2. (i) It is not difficult to deduce that the above result remains valid in the case of a $p$-norm, $\|\cdot\|_{p}, 1<p<\infty$. We do not know whether the converse assertion in Theorem 3.1 (with our regularity assumptions) holds in the cases $p=1$ or $p=\infty$.

(ii) The notions of parametric representation and asymptotic starlikeness can be easily extended to the case of an arbitrary norm in $\mathbb{C}^{n}$. In this case, we may prove that if $f$ has parametric representation, then $f$ is also asymptotically starlike, since the compactness of the analog of the Carathéodory class in $\mathbb{C}^{n}$ remains valid by 4 .

From Theorem 3.1 and Remark 1.3 we deduce the following equivalent formulation of Theorem 3.1 (compare with [16]):

Theorem 3.3. Let $f \in S\left(B^{n}\right)$. Then $f$ is asymptotically starlike if and only if there exists a Loewner chain $f(z, t)$ such that $\left\{e^{-t} f(\cdot, t)\right\}_{t \geq 0}$ is a normal family on $B^{n}$ and $f=f(\cdot, 0)$.

In view of Theorem 3.3. we obtain the following consequence, which shows that the notion of asymptotic starlikeness contains that of spirallikeness of type $\alpha$.

Corollary 3.4. Let $\alpha \in(-\pi / 2, \pi / 2)$ and $f: B^{n} \rightarrow \mathbb{C}^{n}$ be a spirallike mapping of type $\alpha$. Then $f$ is asymptotically starlike. In particular, if $f \in S^{*}\left(B^{n}\right)$, then $f$ is asymptotically starlike.

Proof. Since $f$ is spirallike of type $\alpha, f(z, t)=e^{(1-i a) t} f\left(e^{i a t} z\right)$ is a Loewner chain, where $a=\tan \alpha$. Clearly $\left\{e^{-t} f(\cdot, t)\right\}_{t \geq 0}$ is a normal family on $B^{n}$, and hence $f=f(\cdot, 0) \in S^{0}\left(B^{n}\right)$. Thus $f$ is asymptotically starlike by Theorem 3.3 .

Remark 3.5. Graham, Hamada and Kohr 4] (cf. 11]) proved that there exist spirallike mappings in $\mathbb{C}^{n}$ which do not have parametric representation for $n \geq 2$, i.e. which are not asymptotically starlike. Indeed, it suffices to consider $n=2$ and $f(z)=\left(z_{1}+a z_{2}^{2}, z_{2}\right), z=\left(z_{1}, z_{2}\right) \in B^{2}$. Then for $A(z)=\left(2 z_{1}, z_{2}\right)$, we have

$$
\Re\left\langle[D f(z)]^{-1} A f(z), z\right\rangle=2\left|z_{1}\right|^{2}+\left|z_{2}\right|^{2}>0, z=\left(z_{1}, z_{2}\right) \in B^{2} \backslash\{0\},
$$

and hence $f$ is spirallike with respect to $A$ for all $a \in \mathbb{C}$. However, if $a>2 \sqrt{15}$ and $z_{0}=(0,1 / 2)$, then $\left\|f\left(z_{0}\right)\right\|>2=\frac{\left\|z_{0}\right\|}{\left(1-\left\|z_{0}\right\|\right)^{2}}$. In view of [4, Corollary 2.4] it follows that $f \notin S^{0}\left(B^{2}\right)$; i.e. $f$ is not asymptotically starlike. However, if $n=1$, then $f \in S$ if and only if $f$ is asymptotically starlike. Indeed, $f \in S$ if and only if $f \in S^{0}(U)$ (see [14]). 
It would be interesting to give an answer to the following:

Open Problem 3.6. Find conditions on the operator $A \in L\left(\mathbb{C}^{n}, \mathbb{C}^{n}\right)$ with $m(A)>$ 0 , such that if $f: B^{n} \rightarrow \mathbb{C}^{n}$ is a spirallike mapping with respect to $A$, then $f$ is asymptotically starlike.

Remark 3.7. In a forthcoming paper [5] we shall study the notion of asymptotic spirallikeness, a natural generalization of asymptotic starlikeness. We shall be concerned with the connection between spirallikeness and asymptotic spirallikeness.

We briefly introduce this notion here and the connection with spirallikeness and nonnormalized univalent subordination chains. For details, see [5].

Definition $3.8([5])$. Let $\Omega \subseteq \mathbb{C}^{n}$ be a domain which contains the origin and let $A \in L\left(\mathbb{C}^{n}, \mathbb{C}^{n}\right)$ be such that $m(A)>0$. We say that $\Omega$ is $A$-asymptotically spirallike if there exists a mapping $Q=Q(z, t): \Omega \times[0, \infty) \rightarrow \mathbb{C}^{n}$ which satisfies the following conditions:

(i) $Q(\cdot, t)$ is a holomorphic mapping on $\Omega, Q(0, t)=0, D Q(0, t)=A, t \geq 0$, and the family $\{Q(\cdot, t)\}_{t \geq 0}$ is locally uniformly bounded on $\Omega$.

(ii) $Q(z, \cdot)$ is measurable on $[0, \infty)$ for all $z \in \Omega$.

(iii) The initial value problem

$$
\partial w / \partial t=-Q(w, t) \text { a.e. } t \geq s, w(z, s, s)=z,
$$

has a unique solution $w=w(z, s, t)$ for each $z \in \Omega$ and $s \geq 0$, such that $w(\cdot, s, t)$ is a holomorphic mapping of $\Omega$ into $\Omega$ for $t \geq s, w(z, s, \cdot)$ is locally absolutely continuous on $[s, \infty)$ locally uniformly with respect to $z \in \Omega$ for $s \geq 0$, and $\lim _{t \rightarrow \infty} e^{t A} w(z, 0, t)=$ $z$ locally uniformly on $\Omega$.

A mapping $f \in S\left(B^{n}\right)$ is called $A$-asymptotically spirallike if $f\left(B^{n}\right)$ is an $A$ asymptotically spirallike domain. Further, a mapping $f \in S\left(B^{n}\right)$ is asymptotically spirallike if there exists an operator $A \in L\left(\mathbb{C}^{n}, \mathbb{C}^{n}\right)$ with $m(A)>0$ such that $f$ is $A$-asymptotically spirallike.

Remark 3.9. (i) Any spirallike domain in $\mathbb{C}^{n}$ with respect to a given operator $A$ is $A$-asymptotically spirallike (see [5]). On the other hand, if $A=I_{n}$ in Definition 3.8 , we obtain the usual notion of asymptotic starlikeness.

(ii) We shall prove the following generalization of Theorem 3.1 (see [5]): if $f \in$ $S\left(B^{n}\right)$ and $k_{+}(A)<2 m(A)$, then $f$ is $A$-asymptotically spirallike if and only if there exists a (non-normalized) univalent subordination chain $f(z, t)$ such that $D f(0, t)=$ $e^{t A},\left\{e^{-t A} f(\cdot, t)\right\}_{t \geq 0}$ is a normal family on $B^{n}$ and $f=f(\cdot, 0)$. Here $k_{+}(A)=$ $\lim _{t \rightarrow \infty} \frac{\ln \left\|e^{t A}\right\|}{t}=\max \{\Re \lambda: \lambda \in \sigma(A)\}$, where $\sigma(A)$ is the spectrum of $A$.

(iii) Also, we shall prove that if $f$ is $A$-asymptotically spirallike and $A$ is such that $A+A^{*}=2 I_{n}$, then $f \in S^{0}\left(B^{n}\right)$. Here $A^{*}$ is the adjoint of $A$. In particular, any spirallike mapping with respect to $A$, such that $A+A^{*}=2 I_{n}$, has parametric representation (see [5]). So this is one class of spirallike mappings for which there is an answer to Open Problem 3.6 .

(iv) The class of asymptotically starlike mappings is compact by [8, Theorem 2.9] and Theorem 3.1. Also, if $k_{+}(A)<2 m(A)$, the class of $A$-asymptotically spirallike mappings is compact; however the full class of asymptotically spirallike mappings is not compact in dimension $n \geq 2$ (see [5]). 


\section{ACKNOWLEDGEMENT}

The authors thank the referee for helpful comments and suggestions that improved the paper.

\section{REFERENCES}

[1] H. Cartan, Sur la possibilité d'étendre aux fonctions de plusieurs variables complexes la théorie des fonctions univalentes, 129-155, Note added to P. Montel, Leçons sur les fonctions univalentes ou multivalentes, Gauthier-Villars, Paris, 1933.

[2] M. Elin, S. Reich and D. Shoikhet, Complex dynamical systems and the geometry of domains in Banach spaces, Dissertationes Mathematicae, 427 (2004), 1-62. MR2071666 (2005g:47118)

[3] S. Gong, Convex and starlike mappings in several complex variables, Kluwer Acad. Publ., Dordrecht, 1998. MR.1689825 (2000c:32054)

[4] I. Graham, H. Hamada and G. Kohr, Parametric representation of univalent mappings in several complex variables, Canadian J. Math., 54 (2002), 324-351. MR1892999|(2003b:32018)

[5] I. Graham, H. Hamada, G. Kohr and M. Kohr, Asymptotically spirallike mappings in several complex variables, J. Anal. Math., to appear.

[6] I. Graham, H. Hamada, G. Kohr, T.J. Suffridge, Extension operators for locally univalent mappings, Michigan Math. J., 50 (2002), 37-55. MR.1897032 (2003e:32029)

[7] I. Graham and G. Kohr, Geometric function theory in one and higher dimensions, Marcel Dekker, Inc., New York, 2003. MR2017933 (2004i:32002)

[8] I. Graham, G. Kohr and M. Kohr, Loewner chains and parametric representation in several complex variables, J. Math. Anal. Appl., 281 (2003), 425-438. MR1982664 (2004b:32024)

[9] I. Graham, G. Kohr and M. Kohr, Basic properties of Loewner chains in several complex variables. In: Geometric Function Theory in Several Complex Variables, C.H. FitzGerald and S. Gong, eds., 165-181, World Sci. Publ., 2004. MR2115789 (2005k:32024)

[10] K. Gurganus, $\Phi$-like holomorphic functions in $\mathbb{C}^{n}$ and Banach spaces, Trans. Amer. Math. Soc., 205 (1975), 389-406. MR0374470 (51:10670)

[11] H. Hamada and G. Kohr, Subordination chains and the growth theorem of spirallike mappings, Mathematica (Cluj), 42 (65) (2000), 153-161. MR.1988620(2004c:32043)

[12] T. Liu, The growth theorems and covering theorems for biholomorphic mappings on classical domains, Doctoral Thesis, Univ. Sci. Tech. China, 1989.

[13] J.A. Pfaltzgraff, Subordination chains and univalence of holomorphic mappings in $\mathbb{C}^{n}$, Math. Ann., 210 (1974), 55-68. MR0352510 (50:4997)

[14] C. Pommerenke, Univalent functions, Vandenhoeck \& Ruprecht, Göttingen, 1975. MR.0507768 (58:22526)

[15] T. Poreda, On the univalent holomorphic maps of the unit polydisc in $\mathbb{C}^{n}$ which have the parametric representation. I. The geometrical properties, Ann. Univ. Mariae Curie Sklodowska, Sect. A, 41 (1987), 105-113. MR:1049182 (91m:32021)

[16] T. Poreda, On the univalent holomorphic maps of the unit polydisc in $\mathbb{C}^{n}$ which have the parametric representation. II. The necessary conditions and the sufficient conditions, Ann. Univ. Mariae Curie Sklodowska, Sect. A, 41 (1987), 115-121. MR1049183 (91m:32022)

[17] T. Poreda, On generalized differential equations in Banach spaces, Dissertationes Mathematicae, 310 (1991), 1-50. MR.1104523 (92i:34079)

[18] S. Reich and D. Shoikhet, Nonlinear semigroups, fixed points, and geometry of domains in Banach spaces, Imperial College Press, London, 2005. MR2022955 (2006g:47105)

[19] T.J. Suffridge, The principle of subordination applied to functions of several variables, Pacific J. Math., 33 (1970), 241-248. MR0261040(41:5660)

[20] T.J. Suffridge, Starlike and convex maps in Banach spaces, Pacific J. Math., 46 (1973), 575-589. MR0374914 (51:11110)

[21] T.J. Suffridge, Starlikeness, convexity and other geometric properties of holomorphic maps in higher dimensions, Lecture Notes in Mathematics, 599, 146-159, Springer-Verlag, 1976. MR0450601 (56:8894) 
Department of Mathematics, University of Toronto, Toronto, Ontario M5S 2E4, CANADA

E-mail address: graham@math.toronto.edu

Faculty of Engineering, Kyushu Sangyo University, 3-1 Matsukadai 2-Chome, HigashiKU FUKUOKA 813-8503, JAPAN

E-mail address: h.hamada@ip.kyusan-u.ac.jp

Faculty of Mathematics and Computer Science, Babeş-Bolyai University, 1 M. KogăLNiceanu Str., 400084 Cluj-Napoca, Romania

E-mail address: gkohr@math.ubbcluj.ro

Faculty of Mathematics and Computer Science, Babeş-Bolyai University, 1 M. KogăLNiCEAnu Str., 400084 Cluj-Napoca, Romania

E-mail address: mkohr@math.ubbcluj.ro 